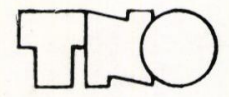

COMMUNICATION NO. 220

CENTRAAL LABORATORIUM T.N.O., Delft

in cooperation with

MATHEMATICAL INSTITUTE, Oxford

\title{
BOND ANGLES IN NITROGEN-CONTAINING HETEROCYCLIC MOLECULES ISOELECTRONIC WITH BENZENE
}

by

C. A. COULSON (Mathematical Inst., Oxford)

and

H. LOOYENGA (Centraal Lab. T.N.O., Delft) 


\section{Preprinted from the Journal of the Chemical Society, November 1965, (1213), pages 6592-6595. \\ 1213. Bond Angles in Nitrogen-containing Heterocyclic Molecules Isoelectronic with Benzene}

By C. A. Coulson and H. Looyenga

It is shown that there is a simple additivity relationship for the bond angles of heterocyclic analogues of benzene with formula $[\mathrm{CH}]_{6-n} \mathrm{~N}_{n}$, in which the replacement of $\mathrm{CH}$ by $\mathrm{N}$ may be treated as if it were a perturbation changing all the angles of the ring by definite amounts.

A simple valence-force field which has three effective parameters is constructed so as to reproduce the observed ring angles in these molecules. It appears that the atoms adjacent to a ring nitrogen are significantly affected by the aza-replacement.

WHEN one of the $\mathrm{CH}$ groups in benzene is replaced by a nitrogen atom, to give pyridine(I), there are certain small changes in the ring angles. The replacement may therefore be treated as a small perturbation. Recently; Kim and Hameka ${ }^{1}$ collected experimental results for the molecules $(\mathrm{I})-(\mathrm{V})$, in which one, two, three, or four such aza-replacements<smiles></smiles>

(I)<smiles>C1CNCCN1</smiles>

(II)<smiles>C1CN2CCC1NC2</smiles>

(III)<smiles>C1NCNCN1</smiles>

(IV)<smiles>C1NNCNN1</smiles>

(V)<smiles>C1CN2C[C@@H]1CN2</smiles>

(VI)

have taken place. The values of the ring angles are shown in the Table. For pyridazine(vi), however, no experimental measurements are available.

Calculated and observed valence angles

\begin{tabular}{|c|c|c|c|c|}
\hline Angle & Exptl. value ${ }^{1}$ & Value from ref. 2 & $\begin{array}{c}\text { Additivity value with } \\
\text { pyridine as basis }\end{array}$ & $\begin{array}{l}\text { Additivity value } \\
\text { using basis (2) }\end{array}$ \\
\hline \multicolumn{5}{|c|}{ Pyridine (I) } \\
\hline $\begin{array}{l}\phi \\
\theta_{1} \\
\theta_{2} \\
\theta_{3}\end{array}$ & $\begin{array}{l}117^{\circ} 10^{\prime} \\
123^{\circ} 53^{\prime} \\
118^{\circ} 28^{\prime} \\
118^{\circ} 20^{\prime}\end{array}$ & $\begin{array}{l}115 \cdot 0^{\circ} \\
124 \cdot 4 \\
119 \cdot 4 \\
117 \cdot 0\end{array}$ & $\begin{array}{l}\left(117 \cdot 2^{\circ}\right) \\
(123 \cdot 9) \\
(118 \cdot 5) \\
(118 \cdot 3)\end{array}$ & $\begin{array}{l}116 \cdot 6^{\circ} \\
124 \cdot 5 \\
118 \cdot 5 \\
118 \cdot 3\end{array}$ \\
\hline \multicolumn{5}{|c|}{ Pyrazine (II) } \\
\hline $\begin{array}{l}\phi \\
\theta\end{array}$ & $\begin{array}{l}115^{\circ} \\
122 \cdot 5\end{array}$ & $\begin{array}{l}112 \cdot 6 \\
123 \cdot 7\end{array}$ & $\begin{array}{l}115 \cdot 2 \\
122 \cdot 4\end{array}$ & $\begin{array}{l}114 \cdot 3 \\
122 \cdot 9\end{array}$ \\
\hline \multicolumn{5}{|c|}{ Pyrimidine (III) } \\
\hline $\begin{array}{c}\phi \\
\theta_{1} \\
\theta_{2}\end{array}$ & & $\begin{array}{l}120 \cdot 1 \\
124 \cdot 0 \\
116 \cdot 9\end{array}$ & $\begin{array}{l}120 \cdot 7 \\
122 \cdot 4 \\
116 \cdot 9\end{array}$ & $\begin{array}{l}121 \cdot 0 \\
122 \cdot 9 \\
116 \cdot 1\end{array}$ \\
\hline \multicolumn{5}{|c|}{$1,3,5$-Triazine (IV) } \\
\hline $\begin{array}{c}\phi \\
\theta_{1} \\
\theta_{2} \\
\theta_{3}\end{array}$ & $\begin{array}{l}115 \\
128 \\
123 \\
116\end{array}$ & $\begin{array}{l}115 \cdot 0 \\
128 \cdot 7 \\
121 \cdot 3 \\
118 \cdot 6\end{array}$ & $\begin{array}{l}115 \cdot 4 \\
127 \cdot 8 \\
122 \cdot 2 \\
117 \cdot 1\end{array}$ & $\begin{array}{l}115 \cdot 0 \\
128 \cdot 9 \\
122 \cdot 2 \\
116 \cdot 8\end{array}$ \\
\hline \multicolumn{5}{|c|}{$1,2,4,5$-Tetrazine (V) } \\
\hline $\begin{array}{l}\phi \\
\theta\end{array}$ & $\begin{array}{l}113 \\
127\end{array}$ & $\begin{array}{l}114 \cdot 4 \\
125 \cdot 6\end{array}$ & $\begin{array}{l}113.9 \\
126.1\end{array}$ & $\begin{array}{l}113 \cdot 2 \\
126 \cdot 6\end{array}$ \\
\hline \multicolumn{5}{|c|}{ Pyridazine (VI) } \\
\hline $\begin{array}{l}\phi \\
\theta\end{array}$ & $\begin{array}{l}116 \\
127\end{array}$ & $\begin{array}{l}116 \cdot 3 \\
127 \cdot 5\end{array}$ & $\begin{array}{l}117 \cdot 6 \\
124 \cdot 8\end{array}$ & $\begin{array}{l}117 \cdot 2 \\
125 \cdot 7\end{array}$ \\
\hline
\end{tabular}

In 1964 one of us ${ }^{2}$ explained these experimental results by means of a simple valenceforce field, in which only one force constant was used. This was for the bending of the 
ring angles. If it was assumed that there was a difference of about $11^{\circ}$ between the natural bond angles of carbon and nitrogen atoms, the whole series of ring angles were predicted as shown in the Table, with a mean error of only $1 \cdot 2^{\circ}$.

The present Paper continues a discussion of these molecules, in two ways. First it shows that there is an additivity relationship for successive aza-replacements, so that the excellent situation described earlier ${ }^{2}$ is less surprising than it might have seemed. Secondly, we look more carefully at the nature of the force-field, on which the shapes of these molecules depend.

The Additivity Relationship.-Since an aza-replacement causes only a small perturbation of ring angles, it would be expected, from the principle of linear additivity of small perturbations, that we could calculate all the bond angles in the molecules (II) - (VI) from a knowledge of the angles in pyridine(I) alone. We are, in effect, supposing that each nitrogen atom introduces its own " pyridine deviations " from $120^{\circ}$ in the ring angles. There will also be changes in bond length, but these are irrelevant for our purpose.

Let us therefore start with the observed pyridine angles. These were obtained by microwave spectroscopy and are likely to be fairly accurate. In terms of the deviations from $120^{\circ}$, these give

$$
\Delta \phi=-3^{\circ} 10^{\prime}, \Delta \theta_{1}=+3^{\circ} 53^{\prime}, \Delta \theta_{2}=-1^{\circ} 28^{\prime}, \Delta \theta_{3}=-1^{\circ} 40^{\prime} .
$$

By the principle of additivity, we should expect, in the case of pyrimidine(III),

$$
\begin{aligned}
& \phi=120^{\circ}-1^{\circ} 28^{\prime}-3^{\circ} 10^{\prime}=115^{\circ} 22^{\prime}, \\
& \theta_{1}=120^{\circ}+3^{\circ} 53^{\prime}+3^{\circ} 53^{\prime}=127^{\circ} 46^{\prime}, \\
& \theta_{2}=120^{\circ}-1^{\circ} 40^{\prime}+3^{\circ} 53^{\prime}=122^{\circ} 13^{\prime}, \\
& \theta_{3}=120^{\circ}-1^{\circ} 28^{\prime}-1^{\circ} 28^{\prime}=117^{\circ} 4^{\prime} .
\end{aligned}
$$

Similar calculations to these give the values shown in the Table. It is clear that agreement with experiment is in general very satisfactory. 1,2,4,5-Tetrazine(v) forms an exception, though even here the greatest error is only just over $2^{\circ}$.

This calculation, in which the mean deviation from experiment is only $0 \cdot 84^{\circ}$, shows very clearly that we are permitted to treat aza-replacement as a small perturbation. There is a sense, therefore, in which we only need to "explain " the pyridine angles; for then the others follow automatically.

Before we attempt this explanation for pyridine, there is another way in which we could treat the additivity situation. One could accept all the experimental values in the Table, and then seek those pyridine-like deviations, $\Delta \phi, \Delta \theta_{1}, \ldots$. which would fit them best. A least-squares fit of the data in the Table leads to the values

$$
\Delta \phi=-3 \cdot 40^{\circ}, \Delta \theta_{1}=+4 \cdot 45^{\circ}, \Delta \theta_{2}=-1 \cdot 60^{\circ}, \Delta \theta_{3}=-2 \cdot 30^{\circ} .
$$

The agreement with $(1)$ is excellent, the differences never exceeding $0 \cdot 7^{\circ}$. There may be some uncertainty about the weighting method used in the above least-squares fit. With a different weighting scheme taking account of the symmetry of some of these molecules, which may make some angles occur twice as often as others, we obtained

$$
\Delta \phi=-3 \cdot 30^{\circ}, \Delta \theta_{1}=+4 \cdot 35^{\circ}, \Delta \theta_{2}=-1 \cdot 45^{\circ}, \Delta \theta_{3}=-1 \cdot 67^{\circ} .
$$

The differences between $(1)$ and $(3)$ are now always less than $\frac{1}{2}^{\circ}$.

Using the pyridine-like deviations (2) and the additivity principle, we compute the bond angles shown in the Table. The mean deviation from experiment is now a little less than $0 \cdot 6^{\circ}$. This is as good as some of the experiments warrant. It must, however, be emphasised that the bond angles of pyridine (determined by microwave methods) are much more reliable than the values for the other molecules (determined by $X$-ray-diffraction). The differences between the first and third columns in the Table are well within the error of an $X$-ray determination. 
The Force Field.-Our discussion so far has been purely phenomenological, and has been set out in order to justify the additivity principle. If we want to find out why the bond angles have their observed values, it follows that we need only concern ourselves with pyridine. If the values for that molecule fit with some proposed force-field, the other molecules will fit also. Our available experimental data are therefore limited to the four distinct angles in pyridine. Since there are two necessary geometrical relationships ${ }^{2}$ involving these angles, we are reduced to two independent experimental quantities. We cannot therefore derive the complete expression for any force-field with more than two parameters. In the previous work ${ }^{2}$ only one effective parameter was introduced; this was the " natural" valence angle of an aromatic nitrogen atom. In order to get a good fit with experiment this was taken to be about $109^{\circ}$, in contrast with the assumed value of $120^{\circ}$ for aromatic carbon.

It is quite possible, however, that the perturbation introduced by aza-replacement, by affecting the polarity of the $\mathrm{C}-\mathrm{N}$ bonds, will change the hybridisation ratios at the carbon atoms. We ought therefore to consider the possibility that there is a different " natural" valence angle for the carbon atoms as well as the nitrogen. This value need not be $120^{\circ}$, and it need not be the same for all the carbon atoms.

We are now dealing with pyridine, and take the notation as in the diagram. As before ${ }^{2}$ we suppose that the force constants for bending at all atoms are the same. We also add a subscript $n\left(e . g ., \phi_{n}, \theta_{1 n}\right)$ to denote a natural valence

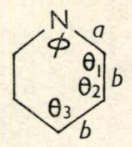
angle. Then the actual angles in pyridine will be such as to minimise the expression $V$, where

$$
2 V=\left(\phi-\phi_{n}\right)^{2}+2\left(\theta_{1}-\theta_{1 n}\right)^{2}+2\left(\theta_{2}-\theta_{2 n}\right)^{2}+\left(\theta_{3}-\theta_{3 n}\right)^{2},
$$

subject to the geometrical relationships

$$
\begin{aligned}
& \mathrm{f}\left(\phi, \theta_{\mathrm{i}}\right) \equiv \phi+2 \theta_{1}+2 \theta_{2}+\theta_{3}-720^{\circ}=0 \\
& \mathrm{~g}\left(\phi, \theta_{\mathrm{i}}\right) \equiv \sin \frac{1}{2} \theta_{3}-\gamma \sin \frac{1}{2} \phi+\sin \left(\theta_{1}+\frac{1}{2} \phi\right)=0
\end{aligned}
$$

where $\gamma=a / b=0.96085$ from experiment. ${ }^{3}$

If we introduce Lagrange undetermined multipliers $(\lambda, \mu)$ this is equivalent to seeking the unrestricted minimum of the expression $(V-\lambda f-\mu g)$. We are led to the set of equations:

$$
\begin{aligned}
& \phi-\phi_{n}-\lambda+\frac{1}{2} \mu \gamma \cos \frac{1}{2} \phi-\frac{1}{2} \mu \cos \left(\theta_{1}+\frac{1}{2} \phi\right)=0, \\
& 2\left(\theta_{1}-\theta_{1 n}\right)-2 \lambda-\mu \cos \left(\theta_{1}+\frac{1}{2} \phi\right)=0, \\
& 2\left(\theta_{2}-\theta_{2 n}\right)-2 \lambda=0 \\
& \theta_{3}-\theta_{3 n}-\lambda-\frac{1}{2} \mu \cos \frac{1}{2} \theta_{3}=0 .
\end{aligned}
$$

The values of $\phi, \theta_{1}, \theta_{2}, \theta_{3}$ are to be those found experimentally. ${ }^{3}$ The unknowns are $\phi_{n}, \theta_{1 n}, \theta_{2 n}, \theta_{3 n}, \lambda$, and $\mu$.

By elimination of $\lambda$ and $\mu$ from the set of equations (4) we are left with two linearly independent equations involving the four natural angles $\phi_{n}, \ldots \theta_{3 n}$. The simplest assumption to make, at this stage, is that the influence of the nitrogen atom extends only to the adjacent carbon atoms. Let us therefore put $\theta_{2 n}=\theta_{3 n}$. The two resulting equations can easily be shown to lead to the results

$$
\phi_{n}-\theta_{1 n}=-7^{\circ} 15^{\prime}, \theta_{1 n}-\theta_{2 n}=+4^{\circ} 58^{\prime} .
$$

It is interesting that these equations represent differences in the natural angles of different atoms. This analysis by itself is not able to predict their absolute values, though, if we decided to put $\theta_{3 n}=\theta_{2 n}=120^{\circ}$, it would lead to the values $\theta_{1 n}=124^{\circ} 56^{\prime}, \phi_{n}=117^{\circ} 41^{\prime}$.

It is important to remember, however, that the " natural " valence angles represented by these values refer only to the atoms as they occur in a pyridine-like situation. They 
may not, therefore, be carried over to other situations, such as in benzene, where the perturbing influence of an aza-nitrogen atom is not present.

The results in (5) may be compared with the earlier single-parameter results: ${ }^{2}$

$$
\phi_{n}-\theta_{1 n}=-11^{\circ}, \theta_{1 n}-\theta_{2 n}=0
$$

It is clear, from both (5) and (6), that the natural valence angle for nitrogen is distinctly less than that for C-1. But (5) suggests that C-1 and C-2 differ considerably. Now, the three-parameter potential function $V$ which we have used is certainly still too simple. We cannot improve it, however, from a consideration of equilibrium structure only. To go any further would require that we calculate and also measure the in-plane vibration frequencies. Until this has been done, we may perhaps be led, from this analysis, to the tentative view that the presence of a ring nitrogen atom exerts a distinct influence on the characteristic valence angles of the carbon atoms adjacent to it.

It would be possible, without great difficulty, to introduce different force constants $\mathrm{k}_{\mathrm{N}}$ and $k_{\mathrm{O}}$ for variations of the bond angles away from their natural values. There is no point in doing so at the moment, without any additional experimental data from which these constants could be obtained.

(C. A. C.) Mathematical Institute, 10 Parks Road, Oxford,

(H. L.) Central Laboratory TNO, Delft.

1 Hojing Kim and H. F. Hameka, J. Amer. Chem. Soc., 1963, 85, 1398.

2 C. A. Coulson, J., 1963, 5893.

3 B. Bak, L. Hansen-Nygaard, and J. Rastrup-Anderson, J. Mol. Spectroscopy, 1958, 2, 361. 